\title{
MONITORING SEVERE ACCIDENTS USING AI TECHNIQUES
}

\author{
YOUNG GYU NO${ }^{1}$, JU HYUN KIM², MAN GYUN NA ${ }^{2 *}$, DONG HYUK LIM ${ }^{3}$, and KWANG-IL AHN ${ }^{1}$ \\ ${ }^{1}$ Korea Atomic Energy Research Institute \\ Dukjin-dong, Yuseong-gu, Daejeon 305-600, Republic of Korea \\ ${ }^{2}$ Department of Nuclear Engineering, Chosun University \\ 375 Seosuk-dong, Dong-gu, Gwangju 501-759, Republic of Korea \\ ${ }^{3}$ Korea Institute of Nuclear Nonproliferation and Control \\ Yuseong-daero, Yuseong-gu, Daejeon 305-348, Republic of Korea \\ ${ }^{*}$ Corresponding author. E-mail : magyna@chosun.ac.kr \\ Invited September14, 2011 \\ Received November 03, 2011 \\ Accepted for Publication February 27, 2012
}

After the Fukushima nuclear accident in 2011, there has been increasing concern regarding severe accidents in nuclear facilities. Severe accident scenarios are difficult for operators to monitor and identify. Therefore, accurate prediction of a severe accident is important in order to manage it appropriately in the unfavorable conditions. In this study, artificial intelligence (AI) techniques, such as support vector classification (SVC), probabilistic neural network (PNN), group method of data handling (GMDH), and fuzzy neural network (FNN), were used to monitor the major transient scenarios of a severe accident caused by three different initiating events, the hot-leg loss of coolant accident (LOCA), the cold-leg LOCA, and the steam generator tube rupture in pressurized water reactors (PWRs). The SVC and PNN models were used for the event classification. The GMDH and FNN models were employed to accurately predict the important timing representing severe accident scenarios. In addition, in order to verify the proposed algorithm, data from a number of numerical simulations were required in order to train the AI techniques due to the shortage of real LOCA data. The data was acquired by performing simulations using the MAAP4 code. The prediction accuracy of the three types of initiating events was sufficiently high to predict severe accident scenarios. Therefore, the AI techniques can be applied successfully in the identification and monitoring of severe accident scenarios in real PWRs.

KEYWORDS : Artificial Intelligence, Severe Accident, GMDH, FNN, MAAP4 Code

\section{INTRODUCTION}

When transient events or accidents occur in nuclear power plants, plant operators are generally provided with only partial information. Even if the operators obtain sufficient information to manage the condition, they often have insufficient time to analyze the accident in urgent situations. In the initial stages of an accident, plant operators will attempt to analyze the abnormal plant states by observing the temporal trends of a few important parameters. However, it is very difficult for operators to predict the progression of an accident by only observing these temporal trends on large display panels in the main control room. Moreover, the operators will be faced with hundreds of instrument readings and alarms that will show some typical patterns for that particular accident, which might increase the level of confusion.

Accident management has increased in importance as a method for preventing the confusion that arises from these conditions and can be accomplished using the operator's high level knowledge of what the initiating events are and where these events have occurred. In the past, there was a model-based approach to diagnosing accidents in nuclear power plants to support operators [1]. Nowadays, the operators' knowledge (database) can be collected using the information obtained from a range of measured data through improvement in the data processing techniques and computing power. When an accident occurs in nuclear power plants, operators will infer and determine the best action for the accident situation using their knowledge, which is a type of database in their brains. This concept is similar to the principles that artificial intelligence (AI) techniques use. Thus, it is expected that the AI techniques will be applicable in predicting severe accident conditions and scenarios. In addition, many studies have examined accident management including accident identification and diagnostics using AI techniques [2]-[11]. AI techniques have also been recently applied to the instrument and system monitoring of nuclear engineering fields [12]-[13].

In order to manage severe accidents in nuclear power 
plants effectively, it is important to predict and identify the accident initiating events within a short time interval after the accident by observing the significant measured signals. Therefore, accurate predictions of the initiating events, such as a loss of coolant accident (LOCA), total loss of feed water (TLOFW), station blackout (SBO), steam generator tube rapture (SGTR), and major plant scenarios for severe accidents, are required in order to manage the severe accidents.

The aim of this study is to develop and verify monitoring techniques for severe accidents in pressurized water reactors (PWRs) using AI methods, such as support vector classification (SVC), probabilistic neural network (PNN), group method of data handling (GMDH), and fuzzy neural networks (FNNs). The SVC and PNN models are used to classify the initiating events, and the GMDH and FNN models are used to monitor the severe accidents. The proposed algorithm was verified using the simulation data from the MAAP4 code [14] for the advanced power reactor 1400 (APR1400), which is an advanced PWR that was developed by the Korea Hydro \& Nuclear Power Company (KHNP). The prediction accuracy of the three types was sufficient to predict severe accidents.

\section{INITIATING EVENT CLASSIFICATION FOR SEVERE ACCIDENTS}

The SVC model that is used widely in classification problems is expected to be able to be applied well to identifying the initiating events that proceed into a severe accident. The SVC model can construct a decision rule to classify the initiating events into one of two classes based on a training data set. Several combined SVC models can solve a general multi-class classification problem that can be extended easily from a binary classification. Therefore, a binary classification is described in this section. In the binary classification of $N$ training data $T=\left\{\mathbf{x}_{i}, y_{i}\right\}_{i=1}^{N}$, where $\mathbf{x}_{i} \in R^{m}$ is the input data vector and $y_{i}$ indicates two classes of $y_{i} \in\{+1,-1\}$. For the case where two classes can be divided linearly, the event classification can be accomplished by defining a hyperplane $(\mathbf{w} \cdot \mathbf{x}+b=0)$ that divides the training data set, where the hyperplane is determined by the coefficient vector $(\mathbf{w})$ and bias $(b)$.

According to the binary classification in Fig. 1, the distance between the two parallel lines of $\mathbf{w} \cdot \mathbf{x}+b=1$ and $\mathbf{w} \cdot \mathbf{x}+b=-1$ is $2 /|\mathbf{w}|$. The separating hyperplane is optimal if the distance between the two parallel lines is the maximum for a given dataset. Therefore, $\mathbf{w}^{T} \mathbf{w}$ should be minimized in order to maximize the distance between the two parallel lines. The generalized optimal separating hyperplane is determined by minimizing the following function:

$$
\Phi(\mathbf{w}, \boldsymbol{\xi})=\frac{1}{2} \mathbf{w}^{T} \mathbf{w}+\lambda \sum_{i=1}^{N} \xi_{i},
$$

subject to the following constraints:

$$
\left\{\begin{array}{l}
y_{i}\left(\mathbf{w} \cdot \mathbf{x}_{i}+b\right) \geq 1-\xi_{i}, \quad i=1,2, \cdots, N \\
\xi_{i} \geq 0, \quad i=1,2, \cdots, N
\end{array}\right.
$$

where

$$
\begin{aligned}
\mathbf{w} & =\left[\begin{array}{llll}
w_{1} & w_{2} & \cdots & w_{m}
\end{array}\right]^{T}, \\
\boldsymbol{\xi} & =\left[\begin{array}{llll}
\xi_{1} & \xi_{2} & \cdots & \xi_{N}
\end{array}\right]^{T} .
\end{aligned}
$$

Even when a hyperplane can separate the data correctly, a method for introducing an additional non-negative parameter $\xi_{i}$ in the second term of Eq. (1) is used to manage the problems associated with misclassifications due to noise in the data. The parameter $\xi_{i}$ is a measure of the misclassification errors. Figure 1 provides an example of a misclassification due to noise in the measured data [9]. The filled triangle and circle indicate the data with the measurement noise. The parameter $\lambda$, which is called a regularization parameter, controls the trade-off between the complex degree of the SVC model and the classification error.

When the linear boundary in the input spaces cannot separate the two classes appropriately, it is possible to create a hyperplane that allows linear separation in a higher dimensional feature space. The SVC models resolve this problem by implicitly mapping the training data into the higher dimensional feature space. That is, the primal space is transformed to a high dimensional feature space using a nonlinear map $\varphi(\mathbf{x})$, as shown in Fig. 2. The function, $\varphi(\mathbf{x})$ is the feature that is mapped nonlinearly from the input space $\mathbf{x}$, where $\boldsymbol{\varphi}=\left[\begin{array}{llll}\phi_{1} & \phi_{2} & \cdots & \phi_{N}\end{array}\right]^{T}$.

Figure 2 shows the hyperplane established in a high dimensional feature space and the nonlinear classification problem converted to a linear classification in the high

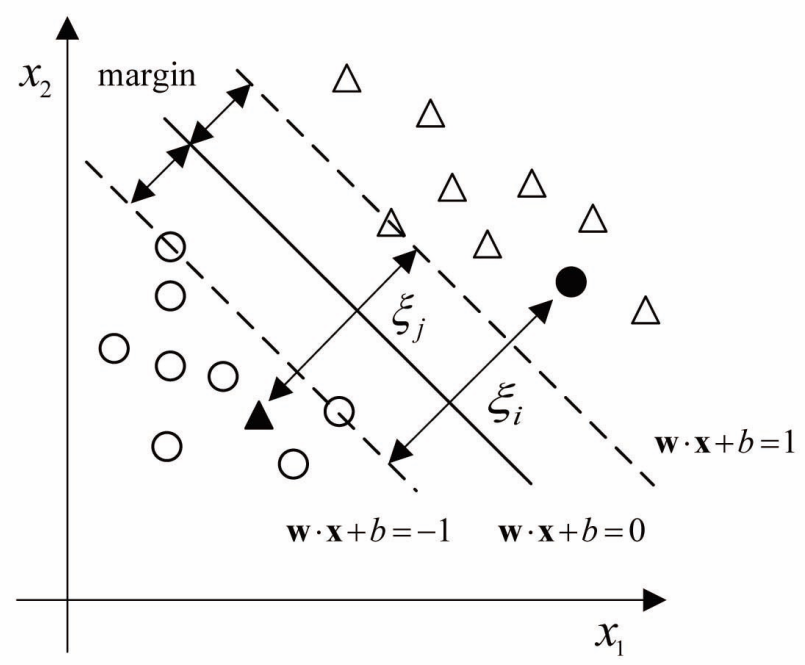

Fig. 1. Example of a Binary Classification and Misclassification Due to Noise in the Measured Data [9]. 
dimensional feature space. The Lagrange multiplier technique and standard quadratic optimization technique can be used to solve the vector $\mathbf{w}$ and bias $b$, and the solution to the convex optimization problem can be expressed using the following equation:

$$
f(x)=\operatorname{sgn}\left(\sum_{i \in S V_{s}} \alpha_{i} y_{i} K\left(\mathbf{x}_{i}, \mathbf{x}\right)+b\right),
$$

where $b^{*}=-\frac{1}{2} \sum_{i=1}^{N} \alpha_{i} \mathbf{y}_{i}\left[K\left(\mathbf{x}_{i}, \mathbf{x}_{r}\right)+K\left(\mathbf{x}_{i}, \mathbf{x}_{s}\right)\right]$ is a bias term and $K\left(\mathbf{x}_{i}, \mathbf{x}\right)=\varphi^{T}\left(\mathbf{x}_{i}\right) \varphi(\mathbf{x})$ is the kernel function. A radial basis function, $K\left(\mathbf{x}_{i}, \mathbf{x}\right)=\exp \left(-\frac{\left(\mathbf{x}-\mathbf{x}_{i}\right)^{T}\left(\mathbf{x}-\mathbf{x}_{i}\right)}{2 \sigma^{2}}\right)$, was used because it exhibited the best performance over a number of simulation applications using different kernel functions conducted in this study.

In this paper, the SVC models were used as non-linear pattern classifiers that categorize the initiating event representing the hot-leg LOCA, cold-leg LOCA, and SGTR using a very short time integration of some selected signals immediately after a reactor scram. The input variables to the SVC models consist of the signals acquired from the reactor coolant system, steam generators, and containment at the nuclear power plant. These signals provide information on the initiating event, such as the hotleg LOCA, cold-leg LOCA, and SGTR. The PNN [16] model provides the initial event classification of a severe accident and was used in a previous study [5]. Therefore, a description of the PNN model was omitted in this paper.

\section{MONITORING OF TRANSIENT SCENARIOS FOR SEVERE ACCIDENTS}

If plant operators follow the accident management guidelines strictly or safety systems work appropriately, a severe accident is unlikely to proceed past the initiating event. However, it is quite difficult for the operators to follow the guidelines and for systems to consistently work well during a plant's lifetime. Therefore, studies of situations leading to a severe accident are required.

In this study, the GMDH [17]-[19] and FNN models were designed to monitor the timing when the following occur: the reactor core is exposed, the core exit temperature (CET) exceeds $1200{ }^{\circ} \mathrm{F}$, which is when severe accident management is normally initiated, and the reactor vessel fails. The proposed accident scenario prediction algorithms are intended to provide plant operators with valuable information, such as the core exposure time and reactor vessel failure time. This prediction problem does not differ from the regression problem to find regression curves using given data (training data). Since the GMDH and FNN models are applied widely to regression problems such as data-based modeling, they can be used effectively to perform severe accident management successfully by predicting the major timings of severe accident scenarios. Therefore, the GMDH and FNN models were developed to predict the important time points that represent major severe accident scenarios.

\subsection{Group Method of Data Handling (GMDH)}

The GMDH is an inductive self-organizing algebraic model and it automatically learns the relationship between the system variables during the training process. Generally, the GMDH model aims to determine the function that most represents a given set of independent variables as dependent variables. The GMDH algorithm uses a data structure similar to multiple regression models in order to improve the prediction accuracy and to select the optimum structure for the model. The acquired data is normally divided into three subsets (training data set, verification data set, test data set) in order to maintain the model normalization and

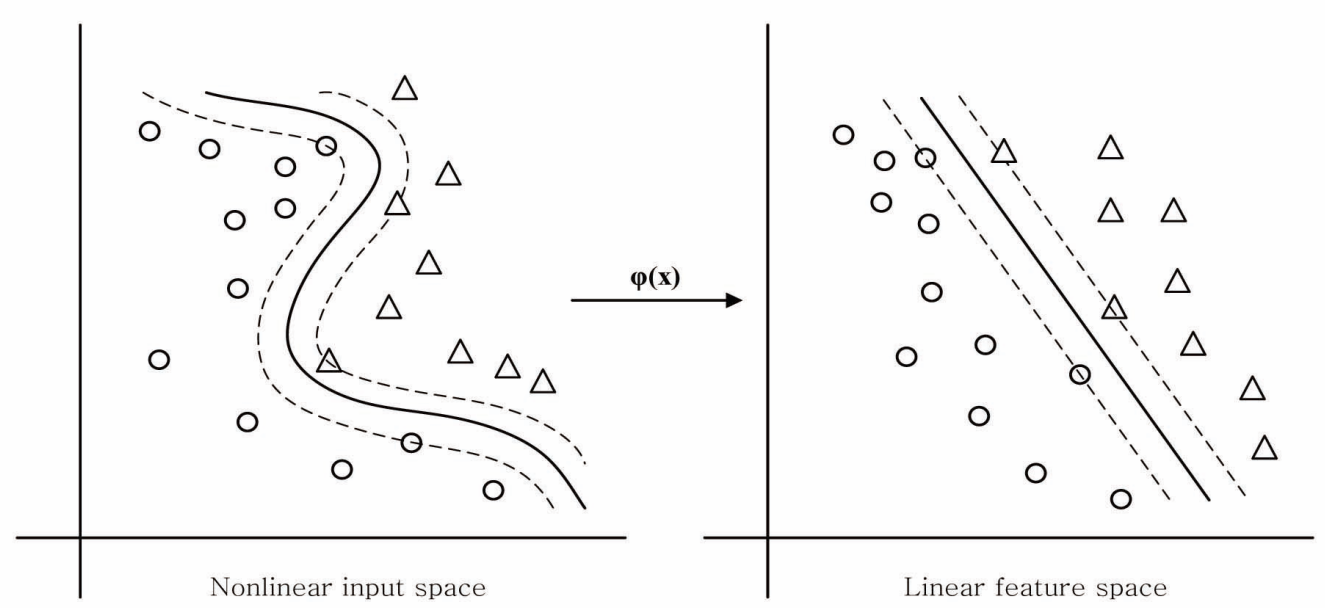

Fig. 2. Mapping to the Linear Feature Space from a Nonlinear Input Space [8]. 
avoid over-fitting via the cross validation. Figure 3 shows the data structure used in the GMDH model. The GMDH employs a self-organizing modeling algorithm that can flexibly choose nonlinear forms of the basic inputs. Figure 4 shows the branch structure of the GMDH algorithm. This begins with the basic inputs at the first level and becomes increasingly complex with an increasing number of layers [20].

The existing GMDH method employs a common format at each step in the continuous approximation [20]-[22]:

$$
y=f\left(x_{i}, x_{j}\right)=A+B x_{i}+C x_{j}+D x_{i}^{2}+E x_{j}^{2}+F x_{i} x_{j} .
$$

The coefficient parameters of the reference function written above $(A, \ldots, F)$ can be solved using a least squares method in an arbitrary pair $\left(x_{i}, x_{j}\right)$ from the independent variables $\mathbf{x}=\left(x_{1}, x_{2}, \ldots, x_{m}\right)$.

The GMDH algorithm uses high-order polynomials in their Kolmogorov-Gabor form [20]-[23]. The KolmogorovGabor form (called Ivakhnenko polynomials) can be expressed as follows:

$$
y=a_{0}+\sum_{i=1}^{m} a_{i} x_{i}+\sum_{i=1}^{m} \sum_{j=1}^{m} a_{i j} x_{i} x_{j}+\sum_{i=1}^{m} \sum_{j=1}^{m} \sum_{k=1}^{m} a_{i j k} x_{i} x_{j} x_{k} \ldots,
$$

where $\mathbf{x}=\left(x_{1}, x_{2}, \ldots, x_{m}\right)$ is an input variable vector and $\mathbf{a}$ $=\left(a_{0}, a_{i}, a_{i j}, a_{i j k}, \ldots\right)$ is the vector of coefficients or the weight of the Kolmogorov-Gabor polynomial.

The GMDH algorithm merges the lower order regression polynomials in each generation in order to reach the next generation. This process continues until the GMDH begins to exhibit over-fitting characteristics in training or it exceeds the specified maximum calculation time [20].

The GMDH algorithm generates and tests all inputoutput combinations in a system. Each element of the system performs the function of two inputs; the elements are the blocks in Fig. 4. The coefficient parameters were decided using a normal least squares method, and the variables of the elements were calculated. The threshold value at each generation determines if the outputs of the elements in a generation are acceptable. In this paper, the threshold at each generation was redefined as the minimum fractional error at the previous generation. The output of an element was eliminated in the current generation when the result was larger than the threshold value. These variables or elements, which are useful for predicting the appropriate output, were used in the next generation. The generations were repeated until satisfactory results were obtained. This process is similar to Darwin's theory, and

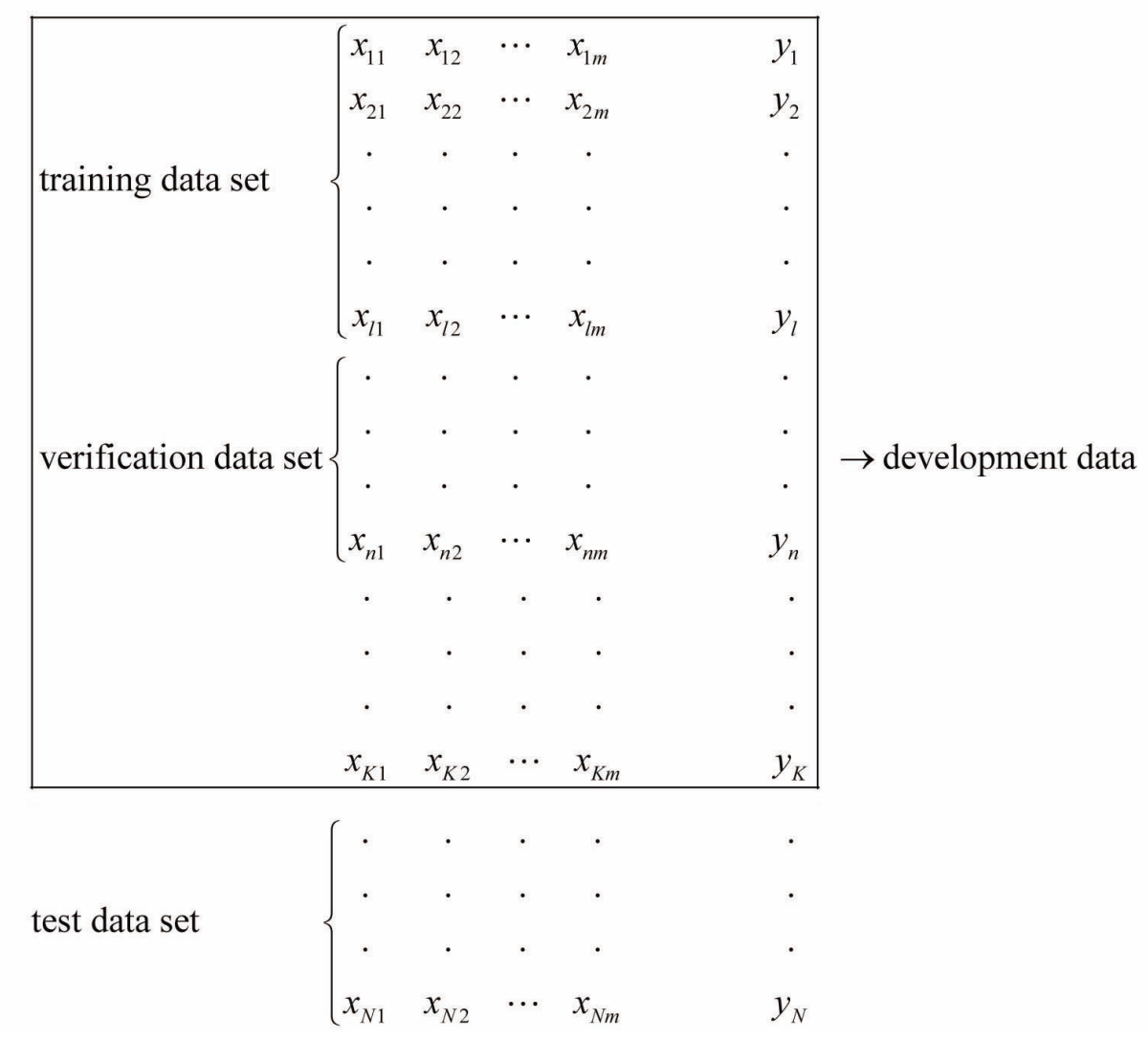

Fig. 3. GMDH Data Structure [17]. 
the detailed key implementation steps are given below [20].

All quadratic regression parameters are stored at the end of the GMDH algorithm procedure, and the estimated coefficients for the high order polynomials are determined by back tracing the GMDH architecture until the original variables, $x_{1}, x_{2}, \ldots, x_{m}$ are reached. In addition, the change in the generations that produce the optimum fit can be expressed as a type of tree known as the Ivakhnenko tree [23].

A GMDH model can be well trained using informative data. The input data representing the NPP operating conditions were organized into net meshes (clusters) (refer to Fig. 5). The centers of the clusters are indicated as ' + ' and are expected to provide more information than the neighboring data. In this paper, a subtractive clustering (SC) scheme [24] was adopted in order to select the cluster centers to be collected for use as the training data. The $\mathrm{SC}$ scheme employs the concept of information potential. The information potential of each data point indicates the information quantity and it is expected that a data point with high information potential has much information. The potential of a data point is defined as being higher when it is surrounded by a greater amount of neighboring data. The input/output data positioned in the cluster centers were used to train the GMDH model.

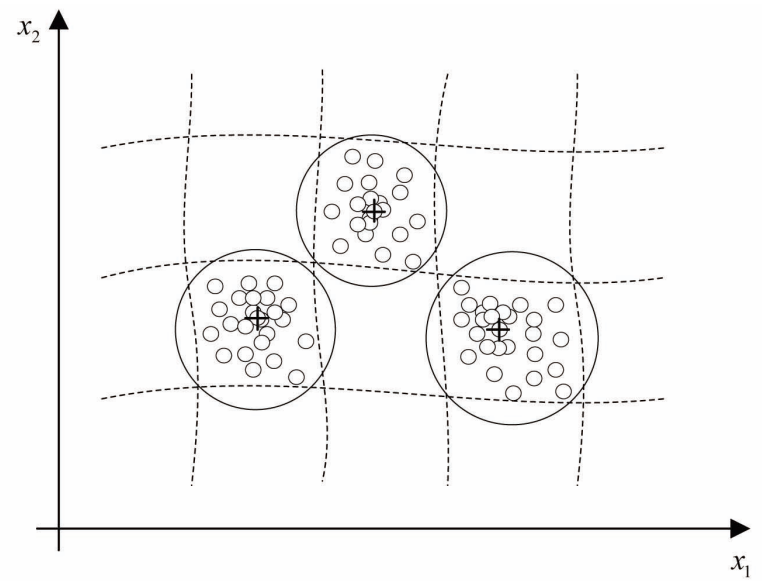

Fig. 5. Net Meshes, Clusters, and Their Centers for a Twodimensional Case.

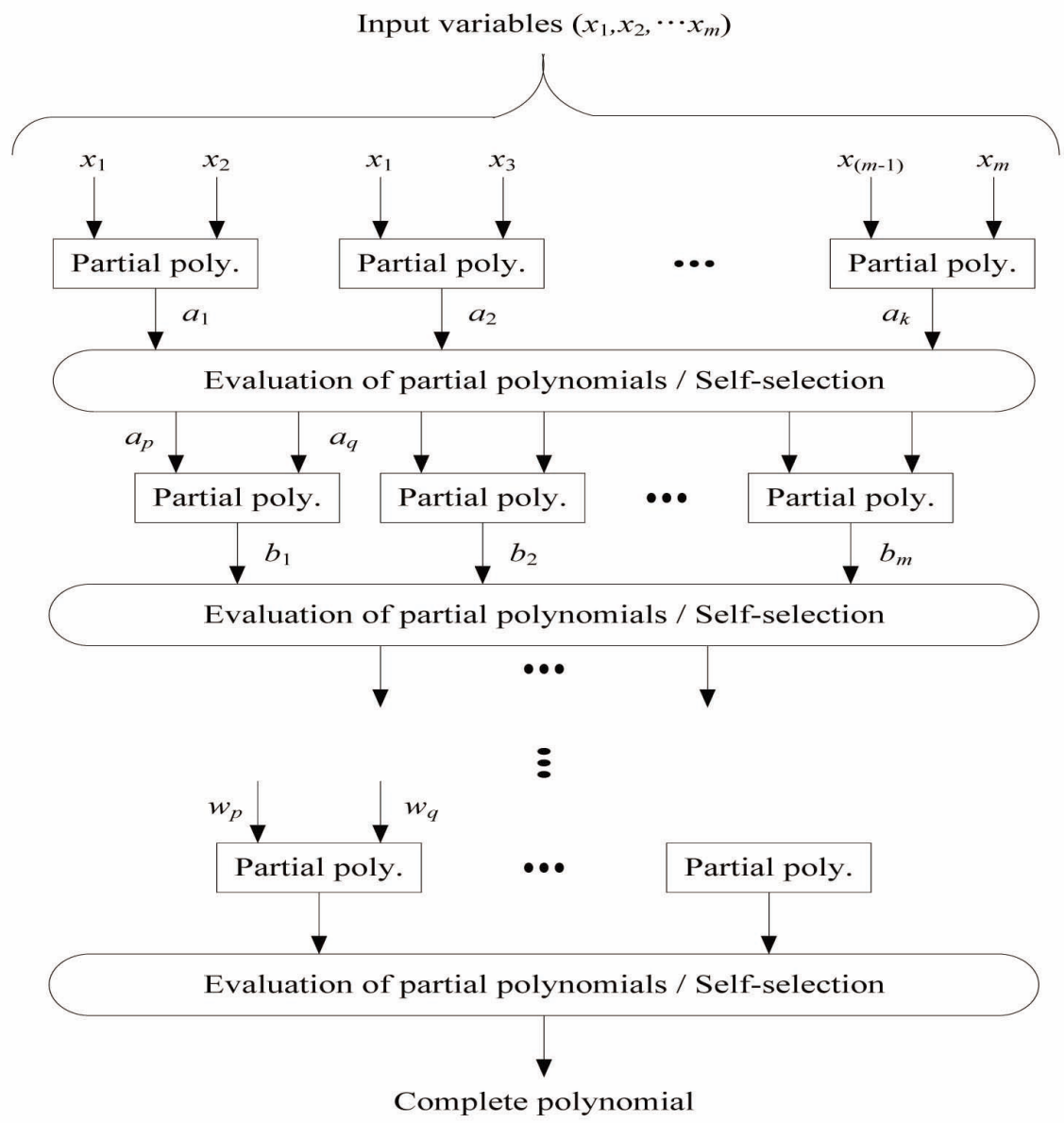

Fig. 4. Branch Structure of the GMDH Model [20]. 


\subsection{Fuzzy Neural Networks (FNNs)}

A fuzzy neural network is a fuzzy inference system equipped with a training algorithm [25]. Because a fuzzy inference system is constructed based on fuzzy if-then rules, linguistic information can be directly incorporated, whereas numerical information is incorporated via training the fuzzy inference system to match the input-output pairs. Therefore, fuzzy neural networks combine linguistic and numerical information (primarily input-output pairs). The main advantages of the fuzzy inference system are the possibility of implementing a rule of thumb experience, intuition, and heuristics, and also that it does not require a mathematical process model.

Instead of employing fuzzy if-then rules that require time-consuming defuzzification calculations of a Mamdani fuzzy inference system [26], the Takagi-Sugeno fuzzy inference system can be described as follows [27]:

$$
\begin{aligned}
& \text { If } x_{1} \text { is } A_{i 1} A N D \cdots A N D x_{m} \text { is } A_{i m} \text {, } \\
& \text { then } \hat{y}^{i} \text { is } f^{i}\left(x_{1}, \cdots, x_{m}\right)
\end{aligned}
$$

where $x_{j}$ is the input variables to the fuzzy neural network ( $j=1,2, \ldots, m ; m=$ number of input variables), $A_{i j}$ is the membership functions for the antecedent of the $i$-th rule and $j$-th input ( $i=1,2, \ldots, n ; n=$ number of rules), and $\hat{y}^{i}$ is the input of the $i$-th rule. Normally, $f^{i}\left(x_{1}, \cdots, x_{m}\right)$ is a polynomial in the input variables, but it can be any function if it can appropriately describe the output of the fuzzy inference system within the fuzzy region specified by the rule antecedent. In this paper, the symmetric Gaussian membership function was used. The output of an arbitrary $i$-th rule, $f^{i}$, consists of the first-order polynomial of inputs, as given in Eq. (7):

$$
f^{i}\left(x_{1}, \cdots, x_{m}\right)=\sum_{j=1}^{m} q_{i j} x_{j}+r_{i},
$$

where $\mathrm{q}_{i j}$ is the weighting value of the $j$-th input on the $i$ th rule output and $r_{i}$ is the bias of the $i$-th rule output. Therefore, the fuzzy inference rule expressed by Eqs. (6) and (7) is called a first-order Takagi-Sugeno fuzzy rule [27].

The output of a fuzzy inference system with $n$ rules is a weighted sum of the consequence of all fuzzy rules. The estimated output of the fuzzy inference system can be expressed as follows:

$$
\hat{y}=\sum_{i=1}^{n} \bar{w}^{i} f^{i}=\mathbf{w}^{T} \mathbf{q},
$$

where

$$
\begin{gathered}
\bar{w}^{i}=\frac{w^{i}}{\sum_{i=1}^{n} w^{i}}, \\
w^{i}=\prod_{j=1}^{m} A_{i j}\left(x_{j}\right), \\
\mathbf{q}=\left[q_{11} \cdots q_{n 1} \cdots \cdots q_{1 m} \cdots q_{n m} r_{1} \cdots r_{n}\right]^{T}, \\
\mathbf{w}=\left[\bar{w}^{1} x_{1} \cdots \bar{w}^{n} x_{1} \cdots \cdots \bar{w}^{1} x_{m} \cdots \bar{w}^{n} x_{m} \bar{w}^{1} \cdots \bar{w}^{n}\right]^{T} .
\end{gathered}
$$

The superscript $i$ indicates that the parameters are related to the $i$-th rule. For a series of $N$ input/output data pairs, the following equation was derived from Eq. (8):

$$
\hat{\mathbf{y}}=\mathbf{W q} \text {, }
$$

where

$$
\begin{aligned}
\hat{\mathbf{y}} & =\left[\begin{array}{llll}
\hat{y}(1) & \hat{y}(2) & \cdots & \hat{y}(N)
\end{array}\right]^{T}, \\
\mathbf{W} & =\left[\begin{array}{llll}
\mathbf{w}(1) & \mathbf{w}(2) & \cdots & \mathbf{w}(N)
\end{array}\right]^{T} .
\end{aligned}
$$

The vector $\mathbf{q}$ is a consequent parameter vector, and the matrix $\mathbf{W}$ consists of the input data and membership function values. A series of outputs of the fuzzy model is represented by the $N \times(m+1) n$ dimensional matrix $\mathbf{W}$ and the $(m+1) n$ dimensional parameter vector $\mathbf{q}$.

\section{VERIFICATION OF THE PROPOSED ALGORITHM}

In order to confirm the proposed algorithm, it is essential to acquire the data required to train the $\mathrm{AI}$ techniques from a number of numerical simulations due to the few real LOCA. The data for a total of 330 accident simulations were performed using the MAAP4 code to acquire the data. The following 15 simulated sensor signals acquired from these simulations were used: core exit temperature, containment pressure and temperature, pressurizer pressure and water level, sump water level, collapsed water level, broken side SG pressure and temperature, broken side SG water level, unbroken side SG pressure and temperature, unbroken side SG water level, refueling water storage tank water level, and containment mole fraction of $\mathrm{H}_{2}$. The containment pressure and temperature are the values measured at the central position of the containment that is located between the operating deck and the polar crane, which is known as the upper compartment below the dome. The APR1400 has two steam generators. The terms 'broken side SG' and 'unbroken side SG' correspond to two steam generators (SGs) connected to a broken hot-leg (or coldleg, SG Tubes (SGTs)) and an unbroken hot-leg (or coldleg, SGTs), respectively. The input variables to the SVC and GMDH models are the time-integrated values of 15 simulated sensor signals as follows:

$$
x_{j}=\int_{t_{s}}^{t_{t}+\Delta t} g_{j}(t) d t, j=1,2, \cdots, 15,
$$

where $g_{j}(t)$ is a specific measured signal, $t_{s}$ is the scram time, and $\Delta t$ is the integration time span.

\subsection{Initiating Event Classification}

A total of 330 accident simulations were classified into three types of initiating events: hot-leg LOCA, coldleg LOCA, and SGTR. In order to confirm the event classification using the proposed algorithm, a total of 330 simulation datasets were divided into model development data and test data. The model development data was used 
to develop the proposed algorithm and the test data was used to test it independently. Therefore, a total of 300 simulation datasets were used to develop the proposed SVC classification algorithm, which consisted of 100 hot-leg LOCAs, 100 cold-leg LOCAs, and 100 SGTRs. The remaining 30 test simulation datasets consisted of 10 hot-leg LOCAs, 10 cold-leg LOCAs, and 10 SGTRs.

In this study, the integrating time span in Eq. (10) for the SVC model was $90 \mathrm{sec}$, which means that the SVC model employs time-integrated signals at $90 \mathrm{sec}$ intervals immediately after a reactor scram. The integrating time span was selected using several numerical simulations of the proposed algorithm in order to minimize the classification error. The two SVC models were trained to categorize the hot-leg LOCA, cold-leg LOCA, and SGTR as $(1,1)$, $(1,-1)$, and $(-1,-1)$, respectively, as shown in Table 1 . The SVC classified the initiating events accurately for both the training and test data. Also, the PNN accurately classified the initiating events of the training and test data. The results of the PNN were abbreviated because the PNN provided the same results as those of the SVC.

\subsection{Accident Scenario Prediction}

Three types of initiating events (hot-leg LOCA, coldleg LOCA, and SGTR) were considered. This study examined the effectiveness of the proposed accidentscenario-prediction algorithm using the GMDH and FNN models in predicting the timing when the following occurred: the reactor core was exposed, the CET exceeded $1200^{\circ} \mathrm{F}$, and the reactor vessel failed. Using the same methods as the event classification, a total of 330 simulation datasets were divided into model development data and test data. A total of 300 simulation datasets were used to develop the GMDH and FNN models, which consisted of 100 hot-leg LOCAs, 100 cold-leg LOCAs, and 100 SGTRs. The remaining 30 test simulation datasets consisted of 10 hot-leg LOCAs, 10 cold-leg LOCAs, and 10 SGTRs. Each 100 development data consisted of 80 training data and 20 verification data. Figure 6 shows the RMS error averaged

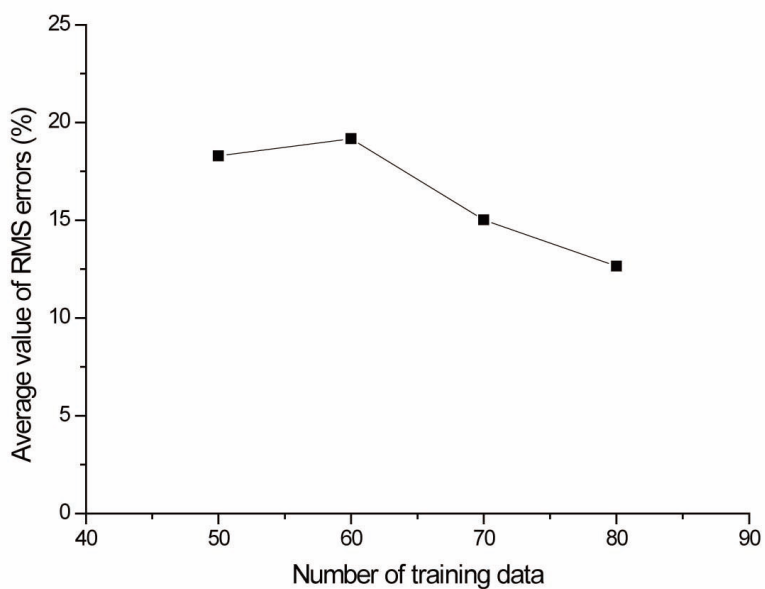

Fig. 6. RMS Error According to the Number of the Training Data (FNN Model).

Table 1. Initiating Event Classification of the Training and Test Data Using SVC.

\begin{tabular}{|c|c|c|c|c|c|c|c|c|c|c|}
\hline & \multirow{3}{*}{$\begin{array}{l}\text { Break size } \\
\quad\left(\mathrm{cm}^{2}\right)\end{array}$} & \multicolumn{3}{|c|}{ Hot-leg LOCA } & \multicolumn{3}{|c|}{ Cold-leg LOCA } & \multicolumn{3}{|c|}{ SGTR } \\
\hline & & \multirow{2}{*}{$\begin{array}{c}\text { Scram } \\
\text { time }(\mathrm{sec})\end{array}$} & \multicolumn{2}{|c|}{ Classified } & \multirow{2}{*}{$\begin{array}{c}\text { Scram } \\
\text { time }(\mathrm{sec})\end{array}$} & \multicolumn{2}{|c|}{ Classified } & \multirow{2}{*}{$\begin{array}{c}\text { Scram } \\
\text { time }(\mathrm{sec})\end{array}$} & \multicolumn{2}{|c|}{ Classified } \\
\hline & & & SVC1 & SVC2 & & SVC1 & SVC2 & & SVC1 & SVC2 \\
\hline \multirow{7}{*}{$\begin{array}{c}100 \\
\text { training } \\
\text { simulations } \\
\text { data }\end{array}$} & 4.50 & 909.91 & 1 & 1 & 568.91 & 1 & -1 & 45.53 & -1 & -1 \\
\hline & 11.15 & 761.91 & 1 & 1 & 224.91 & 1 & -1 & 45.76 & -1 & -1 \\
\hline & 17.80 & 646.91 & 1 & 1 & 137.91 & 1 & -1 & 45.54 & -1 & -1 \\
\hline & $\sim(94)$ & $\sim(94)$ & $\sim(94)$ & $\sim(94)$ & $\sim(94)$ & $\sim(94)$ & $\sim(94)$ & $\sim(94)$ & $\sim(94)$ & $\sim(94)$ \\
\hline & 716.35 & 7.42 & 1 & 1 & 5.98 & 1 & -1 & 7.60 & -1 & -1 \\
\hline & 723.01 & 7.38 & 1 & 1 & 5.95 & 1 & -1 & 7.55 & -1 & -1 \\
\hline & 729.66 & 7.34 & 1 & 1 & 5.92 & 1 & -1 & 7.50 & -1 & -1 \\
\hline \multirow{5}{*}{$\begin{array}{c}10 \text { test } \\
\text { simulations } \\
\text { data }\end{array}$} & 37.76 & 126.91 & 1 & 1 & 64.41 & 1 & -1 & 9.81 & -1 & -1 \\
\hline & 104.29 & 38.99 & 1 & 1 & 23.78 & 1 & -1 & 8.73 & -1 & -1 \\
\hline & $\sim(6)$ & $\sim(6)$ & $\sim(6)$ & $\sim(6)$ & $\sim(6)$ & $\sim(6)$ & $\sim(6)$ & $\sim(6)$ & $\sim(6)$ & $\sim(6)$ \\
\hline & 616.56 & 8.22 & 1 & 1 & 6.46 & 1 & -1 & 7.22 & -1 & -1 \\
\hline & 689.74 & 7.62 & 1 & 1 & 6.12 & 1 & -1 & 7.62 & -1 & -1 \\
\hline
\end{tabular}


for nine cases (three different break locations each using three different event timings) using the FNN model. As shown in this figure, good results were obtained using the 80 training data.

The integrating time span in Eq. (10) depends on the types of input signals and initiating events. The time span was determined using the correlation between the related timing (the output of the SVR and FNN models) and the integrated input signals (Table 2). The correlation coefficient matrices between the output and input signals were calculated every 10 seconds of the integration time span from 30 seconds to 90 seconds, and the integration time span with maximum correlation degree was chosen. This means that the GMDH model can predict these important scenario timings using the initial short time integration of the measured input signals for 30 to 90 seconds immediately after a reactor scram. All 15 signals were not used to estimate the major timings of severe accidents caused by LOCA, because they had little relation with the major timings and it takes considerable time to train and optimize the GMDH and FNN models if too many inputs are used in the models. Many measured signals were first removed from the input signals to the GMDH and FNN models through analysis and graphical plotting of the input ( $\mathrm{x}$ axis) and output (y axis) signals at many data points and through analyzing the relationship between the input and output data by calculating the covariance matrix of all acquired data. In addition, the optimal input selection process was performed using a genetic algorithm. Table 2 lists the selected inputs and integrated time spans according to the input signals and initiating events. Furthermore, the 100 model development data were divided into two data sets: training data (80 data points) and verification data (20 data points). The training data was used to train the GMDH model and the verification data was used to prevent over-fitting.

Table 3 lists the prediction errors for the GMDH model. A comparison of the results of the training and test data revealed a significant difference between the relative and RMS error, because the test data size was too small. However, providing sufficient simulation data can decrease these differences.

Figure 7 shows the important timings in severe accident scenarios that were induced by the initiating event of the hot-leg LOCAs when using the GMDH model. These graphs demonstrate that the proposed algorithm accurately predicts the important timings that represent the major severe accident scenarios, including the predicted timings and their errors. The timing and relative prediction errors are indicated in the left and right vertical axes, respectively. Figure 7(a) shows the target and predicted timings for the reactor core exposure using four input variables (refer to Table 2). In this figure, the relative error represents the percent error of $\left(y_{k}-\hat{y}_{k}\right) / y_{k}$. The large relative error in the test data resulted from the relatively small target value. The predicted value was similar to the target value. It may be possible to reduce the error by training the GMDH model using more training data. Figure 7(b) shows the target and predicted timings for the time when the CET exceeds $1200{ }^{\circ} \mathrm{F}$ using four input variables (refer to Table 2).

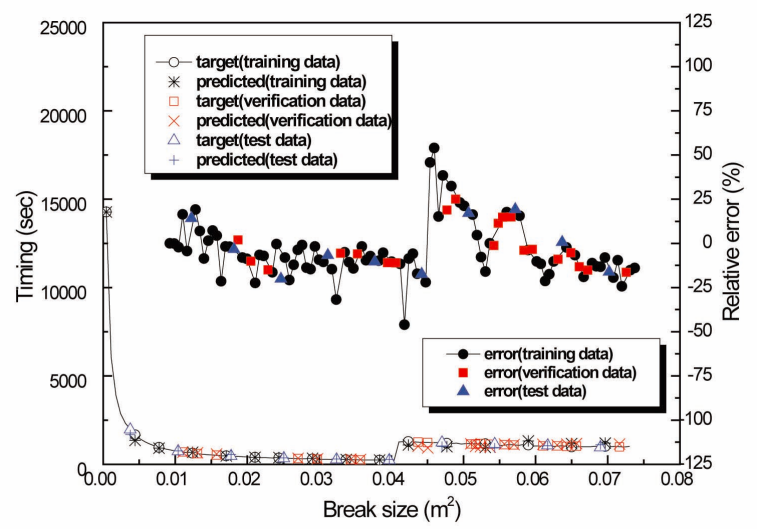

(a) Reactor core exposure time.

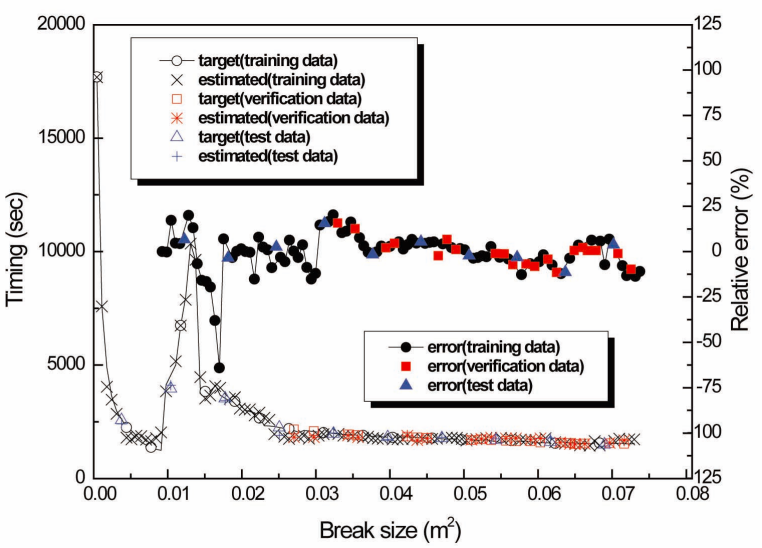

(b) Time when CET exceeds $1200^{\circ} \mathrm{F}$.

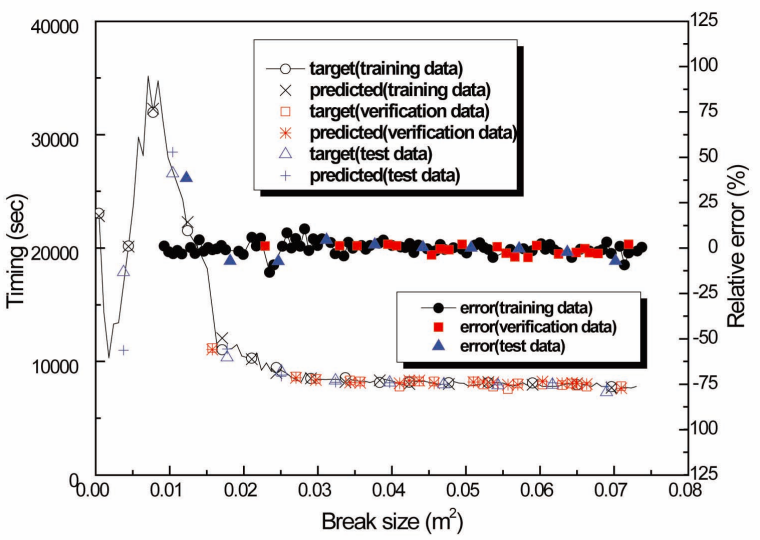

(c) Reactor vessel failure time.

Fig. 7. Important Scenario Timings Due to the Initiating Event of Hot-leg LOCA Using the GMDH Model. 
Figure 7(c) shows the target and predicted timings for the reactor vessel failure time using three input variables (refer to Table 2). Overall, Fig. 7 shows that the proposed GMDH model can accurately predict the important timings

Table 2. Selected Input Variables and Integrating Time Span.

\begin{tabular}{|c|c|c|c|}
\hline Initiating event & Scenario type & Selected inputs & Integrating time span (sec) \\
\hline \multirow{11}{*}{$\begin{array}{l}\text { Hot-leg } \\
\text { LOCA }\end{array}$} & \multirow{4}{*}{ Core exposure time } & Pressurizer water level & 90 \\
\hline & & Collapsed sump water level & 30 \\
\hline & & Collapsed water level & 40 \\
\hline & & Unbroken side SG water temperature & 60 \\
\hline & \multirow{4}{*}{ Time when CET exceeds $1200^{\circ} \mathrm{F}$} & Containment pressurizer & 30 \\
\hline & & Containment gas temperature & 90 \\
\hline & & Pressurizer water level & 50 \\
\hline & & Collapsed water level & 90 \\
\hline & \multirow{3}{*}{ Reactor vessel failure time } & Pressurizer pressure & 30 \\
\hline & & Pressurizer water level & 30 \\
\hline & & Broken side SG water level & 90 \\
\hline \multirow{13}{*}{$\begin{array}{l}\text { Cold-leg } \\
\text { LOCA }\end{array}$} & \multirow{6}{*}{ Core exposure time } & Collapsed sump water level & 30 \\
\hline & & Collapsed water level & 60 \\
\hline & & Broken side SG water level & 30 \\
\hline & & Broken side SG pressure & 60 \\
\hline & & Unbroken side SG water level & 30 \\
\hline & & Unbroken side SG pressure & 70 \\
\hline & \multirow{4}{*}{ Time when CET exceeds $1200^{\circ} \mathrm{F}$} & Containment gas temperature & 40 \\
\hline & & Pressurizer pressure & 90 \\
\hline & & Unbroken side SG water level & 90 \\
\hline & & Broken side SG water temperature & 30 \\
\hline & \multirow{3}{*}{ Reactor vessel failure time } & Containment pressurizer & 90 \\
\hline & & Pressurizer pressure & 30 \\
\hline & & Broken side SG water level & 90 \\
\hline \multirow{9}{*}{ SGTR } & \multirow{3}{*}{ Core exposure time } & Collapsed water level & 90 \\
\hline & & Broken side SG water level & 30 \\
\hline & & Unbroken side SG water level & 90 \\
\hline & \multirow{3}{*}{ Time when CET exceeds $1200^{\circ} \mathrm{F}$} & Core exit temperature & 30 \\
\hline & & Pressurizer pressure & 30 \\
\hline & & Broken side SG water level & 30 \\
\hline & \multirow{3}{*}{ Reactor vessel failure time } & Core exit temperature & 30 \\
\hline & & Pressurizer water level & 30 \\
\hline & & Broken side SG water temperature & 30 \\
\hline
\end{tabular}


for severe accident scenarios initiated by a hot-leg LOCA. The graphs related to the cold-leg LOCA and SGTR were similar to those of the hot-leg LOCA and have thus been omitted from this paper for brevity.

Table 4 lists the prediction errors for the FNN model. Note that the GMDH and FNN models used the same data and conditions. The prediction errors were $11.8743 \%$, averaged from the nine different applications of the GMDH model (refer to Table 3), and $12.6450 \%$ for that of the FNN model. By comparing Tables 3 and 4, the GMDH model is slightly superior to the FNN model in severe accident scenarios.

Table 3. Prediction Errors for the GMDH Model.

\begin{tabular}{|c|c|c|c|c|c|}
\hline \multirow{2}{*}{$\begin{array}{l}\text { Initiating } \\
\text { event }\end{array}$} & \multirow{2}{*}{ Scenario type } & \multicolumn{2}{|c|}{ Training data } & \multicolumn{2}{|c|}{ Test data } \\
\hline & & RMS error (\%) & Relative max. error (\%) & RMS error (\%) & Relative max. error (\%) \\
\hline \multirow{3}{*}{$\begin{array}{l}\text { Hot-leg } \\
\text { LOCA }\end{array}$} & Core exposure time & 16.6385 & 54.0076 & 14.1283 & 20.1601 \\
\hline & $\begin{array}{l}\text { Time when CET } \\
\text { exceeds } 1200^{\circ} \mathrm{F}\end{array}$ & 11.9113 & 64.0588 & 7.0560 & 15.6363 \\
\hline & RV failure time & 3.5284 & 20.1601 & 12.9123 & 38.5437 \\
\hline \multirow{3}{*}{$\begin{array}{l}\text { Cold-leg } \\
\text { LOCA }\end{array}$} & Core exposure time & 11.7598 & 33.1136 & 28.8688 & 73.4936 \\
\hline & $\begin{array}{l}\text { Time when CET } \\
\text { exceeds } 1200^{\circ} \mathrm{F}\end{array}$ & 6.4431 & 37.5857 & 18.3856 & 42.7712 \\
\hline & RV failure time & 3.3921 & 10.9808 & 10.1714 & 30.9587 \\
\hline \multirow{3}{*}{ SGTR } & Core exposure time & 1.4383 & 4.3843 & 1.4742 & 3.5844 \\
\hline & $\begin{array}{l}\text { Time when CET } \\
\text { exceeds } 1200^{\circ} \mathrm{F}\end{array}$ & 1.5897 & 7.0196 & 2.0519 & 5.8674 \\
\hline & RV failure time & 10.9552 & 27.6353 & 11.8209 & 19.4668 \\
\hline
\end{tabular}

Table 4. Prediction Errors for the FNN Model.

\begin{tabular}{|c|c|c|c|c|c|}
\hline \multirow{2}{*}{$\begin{array}{c}\text { Initiating } \\
\text { event }\end{array}$} & \multirow{2}{*}{ Scenario type } & \multicolumn{2}{|c|}{ Training data } & \multicolumn{2}{|c|}{ Test data } \\
\hline & & RMS error $(\%)$ & Relative max. error (\%) & RMS error $(\%)$ & Relative max. error $(\%)$ \\
\hline \multirow{3}{*}{$\begin{array}{l}\text { Hot-leg } \\
\text { LOCA }\end{array}$} & Core exposure time & 17.6697 & 82.2923 & 16.2277 & 40.9958 \\
\hline & $\begin{array}{l}\text { Time when CET } \\
\text { exceeds } 1200^{\circ} \mathrm{F}\end{array}$ & 17.7643 & 63.5521 & 21.9829 & 59.1053 \\
\hline & RV failure time & 4.8928 & 15.0743 & 6.0753 & 14.5531 \\
\hline \multirow{3}{*}{$\begin{array}{l}\text { Cold-leg } \\
\text { LOCA }\end{array}$} & Core exposure time & 38.0543 & 255.4807 & 20.1647 & 56.7208 \\
\hline & $\begin{array}{l}\text { Time when CET } \\
\text { exceeds } 1200^{\circ} \mathrm{F}\end{array}$ & 9.0084 & 51.4812 & 11.6716 & 29.8052 \\
\hline & $\mathrm{RV}$ failure time & 4.7532 & 15.5016 & 19.1405 & 59.2861 \\
\hline \multirow{3}{*}{ SGTR } & Core exposure time & 2.1140 & 5.3297 & 2.4352 & 5.7241 \\
\hline & $\begin{array}{l}\text { Time when CET } \\
\text { exceeds } 1200^{\circ} \mathrm{F}\end{array}$ & 1.7441 & 5.1782 & 3.1486 & 9.5521 \\
\hline & $\mathrm{RV}$ failure time & 11.5379 & 30.8236 & 12.9581 & 26.3952 \\
\hline
\end{tabular}


Figure 8 shows the important timings in severe accident scenarios for the FNN model induced by the initiating event of hot-leg LOCAs. Figure 8(a) shows the target and predicted timings for the reactor core exposure; Fig. 8(b) shows the target and predicted timings for the time

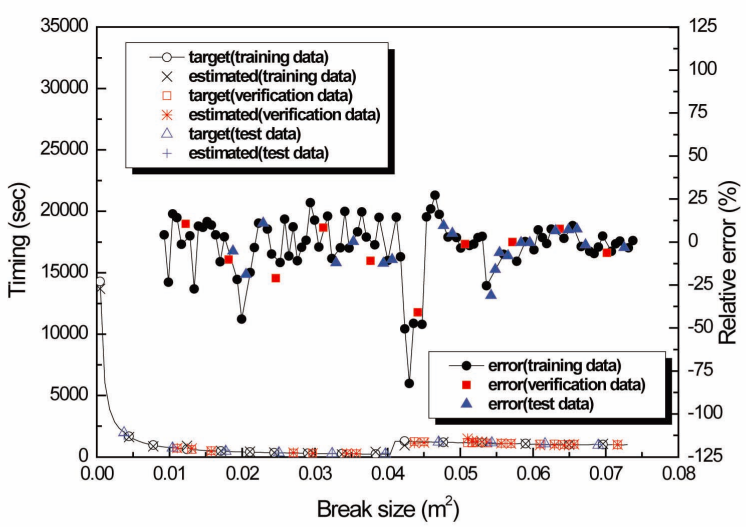

(a) Reactor core exposure time.

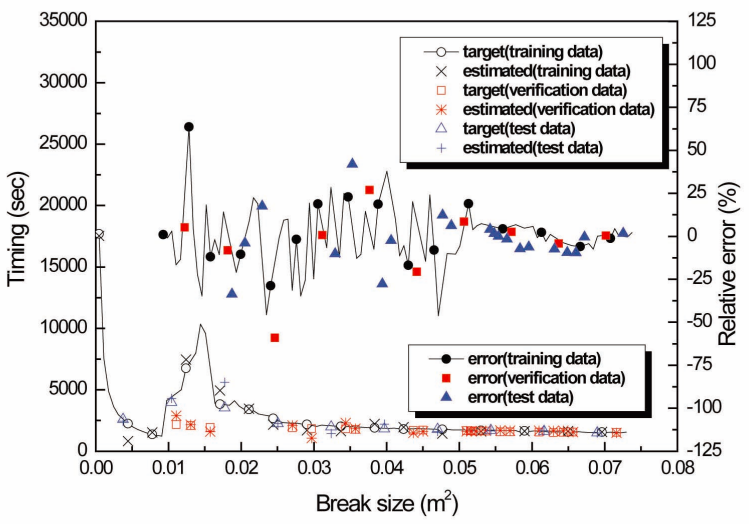

(b) Time when CET exceeds $1200{ }^{\circ} \mathrm{F}$.

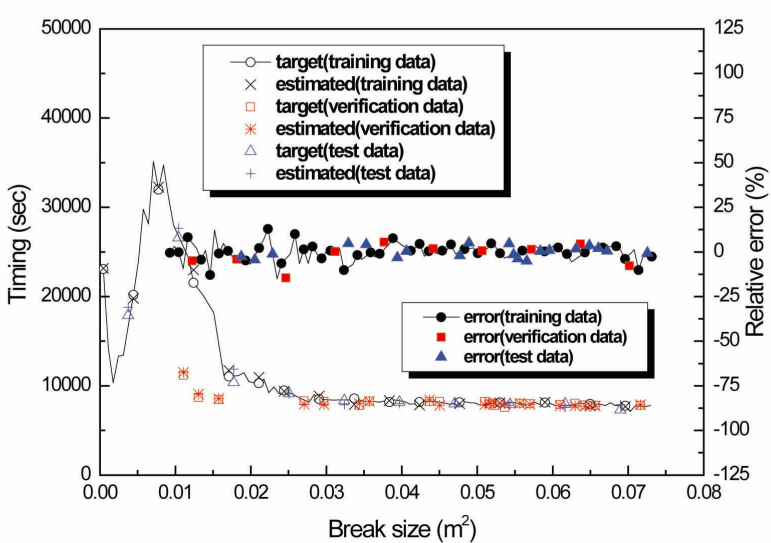

(c) Reactor vessel failure time.

Fig. 8. Important Scenario Timings Due to the Initiating Event of Hot-leg LOCA Using the FNN Model. when the CET exceeds $1200^{\circ} \mathrm{F}$. Figure $8(\mathrm{c})$ presents the target and predicted timings for the reactor vessel failure time. Overall, Fig. 8 shows that the proposed FNN model can accurately predict the important timings for severe accident scenarios that are initiated by a hot-leg LOCA. The graphs related to the cold-leg LOCA and SGTR were similar to the hot-leg LOCA and have thus been omitted from this paper for brevity.

\section{CONCLUSIONS}

In order to effectively manage severe accidents in nuclear power plants, many studies have examined accident management including event identification using artificial intelligence techniques. In this paper, the SVC model was designed to classify the initiating events into three types of categorized events: hot-leg LOCA, cold-leg LOCA, and SGTR. In addition, the GMDH and FNN models were used to predict severe accidents and were developed to predict the important timings representing severe accident scenarios, such as the reactor core exposure time, the time when the CET exceeds $1200^{\circ} \mathrm{F}$, and the reactor vessel failure time due to LOCA. The proposed AI techniques were applied and verified using the data acquired using the MAAP4 code. In addition, more informative data obtained from an SC scheme were used to train the models.

The simulation results demonstrated that the proposed SVC could accurately classify numerous initiating events into three types of categorized events: hot-leg LOCA, coldleg LOCA, and SGTR. In addition, the proposed GMDH and FNN models could predict within approximately $30 \%$ RMS error the important timings representing severe accident scenarios, such as the reactor core exposure time, the time when the CET exceeds $1200^{\circ} \mathrm{F}$, and the reactor vessel failure time due to LOCA. The proposed GMDH model was compared with the FNN model and the GMDH model was found to be slightly superior to the FNN model. Therefore, the SVC and GMDH models can be applied successfully in the identification and prediction of severe accident scenarios in real nuclear power plants.

\section{REFERENCES}

[1] Akio Gofuku, Hidekazu Yoshikawa, Shunsuke Hayashi, Kenji Shimizu, Jiro Wakabayashi, "Diagnostic techniques of a small-break loss-of-coolant accident at a pressurized water reactor plant," Nucl. Technol., vol. 81, pp. 313-332, 1988.

[2] S. W. Cheon and S. H. Chang, "Application of neural networks to a connectionist expert system for transient identification in nuclear power plants," Nucl. Technol., vol. 102, no. 2, pp. 177-191, May 1993.

[ 3 ] Y. Bartal, J. Lin, and R. E. Uhrig, "Nuclear power plant transient diagnostics using artificial neural networks that allow "don't-know" classifications," Nucl. Technol., vol. 110, no. 3, pp. 436-449, June 1995. 
[4] K. Nabeshima, T. Suzudo, T. Ohno, K. Kudo, "Nuclear reactor monitoring with the combination of neural network and expert system," Mathematics and Computers in Simulation, vol. 60, pp. 233-244, 2002.

[ 5 ] M. G. Na, S. M. Lee, S. H. Shin, D. W. Jung, S. P. Kim, J. H. Jeong, and B. C. Lee, "Prediction of major transient scenarios for severe accidents of nuclear power plants," IEEE Trans. Nucl. Sci., vol. 51, no. 2, pp. 313-321, April 2004.

[6] Antonio C.A. Mol, et al., "Neural and genetic-based approaches to nuclear transient identification including 'don't know' response,' Progress in Nuclear Energy, vol. 48, pp. 268-282, 2006.

[ 7 ] T.V. Santosh, et al., "Diagnostic system for identification of accident scenarios in nuclear power plants using artificial neural networks," Reliability Engineering and System Safety, vol. 94, pp. 759-762, 2009.

[8] M. G. Na, W. S. Park, and D. H. Lim, "Detection and diagnostics of loss of coolant accidents using support vector machines," IEEE Trans. Nucl. Sci., vol. 55, no. 1, pp. 628-636, Feb. 2008.

[9] S. H. Lee, Y. G. No, M. G. Na, K.-I. Ahn and S.-Y. Park, "Diagnostics of loss of coolant accidents using SVC and GMDH models," IEEE Trans. Nucl. Sci., vol. 58, no. 1, pp. 267-276, Feb. 2011.

[10] Paolo F. Fantoni, "Experiences and applications of PEANO for online monitoring in power plants," Progress in Nucl. Energy, vol. 46, pp. 206-225, 2005.

[11] Jamie Garvey, Dustin Garvey, Rebecca Seibert and J. Wesley Hines, "Validation of on-line monitoring techniques to nuclear plant data," Nucl. Eng. Technol., vol. 39 no. 2 pp. 149-158, 2007

[12] I.-Y.Seo, B.-N. Ha, S.-W. Lee, C.-H. Shin, and S.-J. Kim, "Principal components based support vector regression model for on-line instrument calibration monitoring in NPPs," Nucl. Eng. Technol., vol. 42, no. 2, pp. 219-230, Apr. 2010.

[13] E. Zio and R. Bazzo, "Optimization of the test intervals of a nuclear safety system by genetic algorithms, solution clustering and fuzzy preference assignment," Nucl. Eng. Technol., vol. 42, no. 4, pp. 414-425, Aug. 2010.

[14] R. E. Henry et al., MAAP4 - Modular Accident Analysis Program for LWR Power Plants, User's Manual. Burr
Ridge, IL: Fauske, vol. 1-4, 1990.

[15] B.-S. Yang, W.-W. Hwang, M.-H. Ko, and S.-J. Lee, "Cavitation detection of butterfly valve using support vector machines," J. Sound Vibr., vol. 287, nos. 1-2, pp. 25-43, Oct. 2005.

[16] D. F. Specht, "Probabilistic neural networks," Neural Networks, vol.3, no. 1, pp. 109-118, 1990.

[17] A. G. Ivakhnenko, "The group method of data handling; a rival of method of stochastic approximation," Soviet Automatic Control, vol. 1, no. 3, pp. 43-55, 1968.

[18] M. C. Acock and Y. A. Pachepsky, "Estimating missing weather data for agricultural simulations using group method of data handling," J. Applied Meteorology, vol. 39, no. 7, pp. 1176-1184, 2000.

[19] T. Kondo, A. S. Pandya, "GMDH-type neural network algorithm with sigmoid function," Intl. J. KnowledgeBased Engineering Systems, vol. 7, no. 4, pp. 198-205, 2003.

[20] S. J. Farlow, Self-Organizing Methods in Modeling: GMDH Type Algorithms. Marcel Dekker, New York, 1984.

[21] C. R. Hild, "Development of The Group Method of Data Handling With Information-based Model Evaluation Criteria: A New Approach to Statistical Modeling," Ph.D. Dissertation, Univ. Tennessee, Knoxville, 1998.

[22] P. B. Ferreira and B. R. Upadhyaya, Incipient Fault Detection and Isolation of Sensors and Field Devices, Nuclear Engineering Dept., Univ. Tennessee, Knoxville, UTNE/BRU/99-02, December 1999.

[23] A. G. Ivakhnenko, "Polynomial theory of complex systems", IEEE Trans. Syst. Man \& Cybern, SMC-1, pp. 364-378, 1971

[24] S. L. Chiu, "Fuzzy model identification based on cluster estimation,” J. Intell. Fuzzy Systems, vol. 2, pp. 267-278, 1994

[25] Jang, J.-S. R., “ANFIS: Adaptive-network-based fuzzy inference systems," IEEE Transactions on Systems, Man, and Cybernetics, vol. 23, pp. 665-685, 1993.

[26] Mamdani, E.H. and Assilian, S., "An experiment in linguistic synthesis with a fuzzy logic controller," Int. J. Man-Machine Studies, vol. 7, pp. 1-13, 1975.

[27] Takagi, T. and Sugeno, M., "Fuzzy identification of systems and its applications to modeling and control," IEEE Trans. System, Man, Cybern., vol. 1, pp. 116-132, 1985. 